\title{
ON VALUE REGIONS OF CONTINUED FRACTIONS
}

\author{
WALTER LEIGHTON AND W. J. THRON
}

The results of this paper are contained in the following theorem.

THEOREM 1. If the elements $a_{1}, a_{2}, \cdots$ of the continued fraction

$$
1+\frac{a_{1}}{1}+\frac{a_{2}}{1}+\cdots
$$

lie in the parabola

$$
\rho \leqq \frac{2 d(1-d)}{1-\cos \theta}, \quad 1 / 2 \leqq d<1,
$$

then the approximants of the continued fraction (1) lie in the hyperbolic region

$$
R>\frac{2 d(1-d)}{1-2 d+\cos \phi}, \quad-\beta<\phi<\beta,
$$

where $\beta=\operatorname{arc} \cos (2 d-1)$. If $z$ is any value on the boundary of the region (3), there exists one and only one continued fraction of the form (1), with elements in the parabola (2), converging to $z$, namely: 1

$$
1+\frac{a}{1}+\frac{\bar{a}}{1}+\frac{a}{1}+\cdots
$$

where $a=(z-1) \bar{z}$ is a value on the boundary of the parabola (2).

For the case $d=1 / 2$, Scott and Wall [3] determined the value region of the approximants and Paydon [1] established the uniqueness property of (4) for that case.

A convergence criterion due to Scott and Wall [2] insures the convergence of the continued fraction (1) if in addition to the conditions of Theorem 1 it is required that the series $\sum\left|b_{n}\right|$ diverges, where $b_{1}=1 / a_{1}, b_{n+1}=1 / a_{n+1} b_{n}$. The value of such a continued fraction must lie in the closure of the region (3). Finally it follows from Theorem 1 that all values in the region (3) are assumed by a continued fraction of the form (4). The following result is now seen to be a consequence of Theorem 1 .

Presented to the Society, April 11, 1942; received by the editors December 7, 1941, and, in revised form, March 27, 1942.

${ }^{1}$ Here and elsewhere in the paper a bar over a number means the complex conjugate of the number. 
THEOREM 2. If the elements $a_{n}(n \geqq 1)$ of the continued fraction (1) lie in the parabola (2) and if in addition $\sum\left|b_{n}\right|$ diverges, where $b_{1}=1 / a_{1}$, $b_{n+1}=1 / a_{n+1} b_{n}$, the continued fraction (1) converges, and its value lies in the closure of the hyperbolic region (3). Further, every value in (3) is taken on by at least one convergent continued fraction (1) with elements in (2).

We now prove the first part of Theorem 1, namely, that the approximants of (1) lie in the region (3) if the elements of the continued fraction lie in the parabola (2). We first note, that if $z$ lies in (3) then $w=r e^{i \psi}=z-1$ satisfies the relation

$$
\begin{array}{lr}
r<\frac{-2 d(1-d)}{1-2 d+\cos \psi}, & \beta<\psi<2 \pi-\beta ; \\
r<\infty, & -\beta<\psi<\beta .
\end{array}
$$

The proof is by induction. $A_{1} / B_{1}-1=a_{1}$ satisfies relation (5), if $a_{1}$ lies in the parabola (2), as

$$
\frac{2 d(1-d)}{1-\cos \phi} \leqq \frac{-2 d(1-d)}{1-2 d+\cos \phi}
$$

for $\beta<\phi<2 \pi-\beta$. Hence $A_{1} / B_{1}$ lies in the region (4). To complete the proof it is sufficient to show that $w=z-1=a / z^{\prime}$ lies in the region defined by relation (5) if $a$ and $z^{\prime}$ are any arbitrary complex numbers in the regions (2) and (3), respectively. To this end we prove the following lemma.

Lemma. The number

$$
w=\frac{a}{z}=\frac{\rho e^{i \phi}}{R e^{i \theta}}=\frac{\rho}{R} e^{i(\phi-\theta)}
$$

satisfies relation (5), if a and $z$ are arbitrary complex numbers in the regions defined by relations (2) and (3), respectively.

We shall choose $a$ and $z$ in such a way, that they maximize $|w|$ for any arbitrary fixed arg $w=\alpha, \beta<\alpha<2 \pi-\beta$. It is clear that the numbers $a$ and $z$ must then be chosen on the boundaries of the regions over which they are allowed to vary, and we then have

$$
|w|=\frac{\rho}{R}=\frac{1-2 d+\cos \theta}{1-\cos \phi}, \quad \phi-\theta=\alpha .
$$

It follows from elementary considerations that if $|w|$ is to be a maximum, $\theta$ must satisfy the relation 


$$
\sin \theta+\sin \alpha+(1-2 d) \sin (\alpha+\theta)=0 .
$$

This is equivalent to

$$
2 \sin \frac{\alpha+\theta}{2}\left[\cos \frac{\alpha-\theta}{2}+(1-2 d) \cos \frac{\alpha+\theta}{2}\right]=0 .
$$

The solution $\theta=-\alpha$ is excluded since the ranges of $\alpha$ and $-\theta$ have no values in common. It remains to find the value of $|w|$ when

$$
\cos \frac{\alpha-\theta}{2}+(1-2 d) \cos \frac{\alpha+\theta}{2}=0 .
$$

We shall show that (6) is equivalent to the two relations $|w|$ $=-2 d(1-d) /(1-2 d+\cos \alpha)$ and $z=1+\bar{w}$. If these two relations are satisfied the sine law leads to

$$
\frac{\sin (\pi-\alpha)}{\sin (-\theta)}=\frac{\sin \alpha}{-\sin \theta}=-\frac{1-2 d+\cos \alpha}{1-2 d+\cos \theta} .
$$

This gives

$$
(1-2 d)(\sin \alpha-\sin \theta)+\sin (\alpha-\theta)=0,
$$

or

$$
2 \sin \frac{\alpha-\theta}{2}\left[\cos \frac{\alpha-\theta}{2}+(1-2 d) \cos \frac{\alpha+\theta}{2}\right]=0
$$

Since $\theta$ cannot equal $\alpha$, the only solution of this equation is that of equation (6). Thus the lemma is proved.

This completes the proof of the first part of Theorem 1. It remains to show that any $z$ on the boundary of (3) is uniquely expressible as a continued fraction of the form (4).

From the proof of the lemma it follows that $a_{1}$ must lie on the boundary of (2) for $z$ to lie on the boundary of (3). It was further shown that

$$
z=1+\frac{a_{1}}{1}+\frac{a_{2}}{1}+\cdots
$$

lies on the boundary only if

$$
\bar{z}=1+\frac{a_{2}}{1}+\frac{a_{3}}{1}+\cdots ;
$$

but then $\bar{z}$ lies on the boundary and hence we must have 


$$
z=1+\frac{a_{2 n-1}}{\bar{z}}, \quad \bar{z}=1+\frac{a_{2 n}}{z}, \quad n \geqq 1 \text {. }
$$

This gives

$$
\begin{aligned}
a_{2 n-1} & =(z-1) \bar{z} \\
a_{2 n} & =(\bar{z}-1) z=\bar{a}_{2 n-1},
\end{aligned}
$$

and it is easily seen that all $a_{n}$ lie on the boundary of the parabola. The theorem is now completely proved.

\section{BIBLIOGRAPHY}

1. J. F. Paydon, Convergence regions and value regions for continued fractions, this Bulletin, abstract 47-11-473.

2. W. T. Scott and H. S. Wall, A convergence theorem for continued fractions, Transactions of this Society, vol. 47 (1940), pp. 155-172.

3. - Value regions for continued fractions, this Bulletin, vol. 47 (1941), pp. $580-585$.

The Rice Institute

\section{A TABLE OF COEFFICIENTS FOR NUMERICAL DIFFERENTIATION}

ARNOLD N. LOWAN, HERBERT E. SALZER AND ABRAHAM HILLMAN ${ }^{1}$

The following table lists the coefficients $A_{m, s}$ for $m=1,2, \cdots, 20$ and $s=m, \cdots, 20$ in Markoff's formula for the $m$ th derivative in terms of advancing differences, namely

$$
\omega^{m} f^{(m)}(x)=\sum_{s=m}^{n-1}(-1)^{m+s} A_{m, s} \Delta^{s} f(x)+(-1)^{m+n} \omega^{n} A_{m, n} f^{(n)}(\xi) .
$$

In this formula $\omega$ is the tabular interval and

$$
A_{m, s}=(-1)^{m+s} m B_{s-m}^{(s)} / s(s-m) !
$$

and $B_{s-m}^{(s)}$ is the $(s-m)$ th Bernoulli number of the $s$ th order.

Presented to the Society, April 4, 1942 under the title Coefficients of differences in the expansion of derivatives in terms of advancing differences; received by the editors March 7, 1942.

${ }^{1}$ The results reported here were obtained in the course of the work done by the Mathematical Tables Project, Work Projects Administration, New York City. 